\title{
ANIMAL SEARCH STRATEGIES: A QUANTITATIVE RANDOM-WALK ANALYSIS
}

\author{
Frederic Bartumeus, ${ }^{1,2,5}$ M. G. E. Da Luz ${ }^{3}$ G. M. Viswanathan, ${ }^{4}$ And J. Catalan ${ }^{1}$ \\ ${ }^{1}$ Centre d'Estudis Avançats de Blanes (CEAB), CSIC, Accés a la Cala Sant Francesc, 17300 Blanes (Girona), Spain \\ ${ }^{2}$ ICREA-Complex Systems Research Laboratory, Universitat Pompeu Fabra C/ Dr. Aiguader 8008003 Barcelona, Spain \\ ${ }^{3}$ Departamento de Física, Universidade Federal do Paraná, 81531-990 Curitiba-PR, Brazil \\ ${ }^{4}$ Departamento de Física, Universidade Federal de Alagoas, 57072-970, Maceió-AL, Brazil
}

Abstract. Recent advances in spatial ecology have improved our understanding of the role of large-scale animal movements. However, an unsolved problem concerns the inherent stochasticity involved in many animal search displacements and its possible adaptive value. When animals have no information about where targets (i.e., resource patches, mates, etc.) are located, different random search strategies may provide different chances to find them. Assuming random-walk models as a necessary tool to understand how animals face such environmental uncertainty, we analyze the statistical differences between two random-walk models commonly used to fit animal movement data, the Lévy walks and the correlated random walks, and we quantify their efficiencies (i.e., the number of targets found in relation to total displacement) within a random search context. Correlated random-walk properties (i.e., scale-finite correlations) may be interpreted as the by-product of locally scanning mechanisms. Lévy walks, instead, have fundamental properties (i.e., super-diffusivity and scale invariance) that allow a higher efficiency in random search scenarios. Specific biological mechanisms related to how animals punctuate their movement with sudden reorientations in a random search would be sufficient to sustain Lévy walk properties. Furthermore, we investigate a new model (the Lévy-modulated correlated random walk) that combines the properties of correlated and Lévy walks. This model shows that Lévy walk properties are robust to any behavioral mechanism providing short-range correlations in the walk. We propose that some animals may have evolved the ability of performing Lévy walks as adaptive strategies in order to face search uncertainties.

Key words: correlated random walks; foraging theory; Lévy walks; random search strategies.

\section{INTRODUCTION}

Standard methods in spatial ecology consider Brownian motion and Fickian diffusion as two basic properties of animal movement at the long-term limit (i.e., large spatial scales and long temporal scales). Thus, it is assumed that animal movements can be modeled (at the long-term limit) as uncorrelated random walks (Okubo 1980, Berg 1983). The problem of uncorrelated random walks is that they do not account for directional persistence in the movement (i.e., the tendency by animals to continue moving in the same direction). Such limitation was overcome with two different types of random walks, correlated random walks (CRWs) and Lévy walks (LWs).

CRWs appeared in ecology from the analysis of short and middle-scaled animal movement data. Experiments with ants, beetles, and butterflies were performed in less than $25-\mathrm{m}^{2}$ arenas, or otherwise, in their natural environments, and usually last less than an hour (e.g., Bovet and Benhamou 1988, Turchin 1991, Crist et al. 1992). From these studies, ecologists promptly became

Manuscript received 2 December 2004; revised 20 April 2005; accepted 27 April 2005. Corresponding Editor: J. Huisman.

${ }^{5}$ E-mail: fbartu@ceab.csic.es aware of the necessity of adding directional persistence into pure random walks to reproduce realistic animal movements (Kareiva and Shigesada 1983, Bovet and Benhamou 1988). More recently, the mathematical properties of CRWs were used to explore the link between individual animal movements and populationlevel spatial patterns (Turchin 1991, 1998). Further studies have considered the relative straightness of the CRW (i.e., degree of directionality; [Haefner and Crist 1994], or sinuosity, [Bovet and Benhamou 1991, Bovet and Bovet 1993, Benhamou 2004]) as relevant properties characterizing animal movement.

The analysis of animal movement at larger spatial scales or at longer temporal scales has given rise to a new category of random-walk models known as Lévy walks (Levandowsky et al. 1988a, Viswanathan et al. 1996). Animal paths involving large spatial or temporal scales (i.e., large-scale animal movement), turn out to be a combination of "walk clusters" with long travels between them. The heterogeneous multiscale-like sampling pattern generated by such paths are closely related to fractal geometries (Mandelbrot 1977) and better modeled by random walks with Lévy statistics. LWs have their origin in the field of statistical mechanics and find wide application in physics (Shlesinger et al. 
1995, Klafter et al. 1996, Weeks and Swinney 1998) and natural sciences such as geology and biology (Metzler and Klafter 2004). Although they have only recently gained attention in optimal foraging theory (Viswanathan et al. 1996, 1999), they appeared in an ecological context around the same decade as CRWs. The first mention of Lévy walks as animal search strategies can be found in Shlesinger and Klafter (1986:283). After that, Lévy walks were formally considered by plankton ecologists (Levandowsky et al. 1988 $a, b$, Klafter et al. 1989).

CRW and LW models have been adjusted successfully to a wide range of empirical data (CRWs [Kareiva and Shigesada 1983, Bovet and Benhamou 1988, Turchin 1991, Crist et al. 1992, Johnson et al. 1992, Bergman et al. 2000], LWs [Viswanathan et al. 1996, Levandowsky et al. 1997, Atkinson et al. 2002, Bartumeus et al. 2003, Ramos-Fernández et al. 2004]). Recent works have introduced the idea of hierarchical scale adjustments on animal displacements (Fritz et al. 2003), and have fitted field data of specific species (Marell et al. 2002, Austin et al. 2004) by using both models. All these studies have shown that CRWs and LWs can be used as fitting procedures to analyze animal movement. Nevertheless, there is a lack of an explicative framework for such an approach, which severely limits the biological interpretation of the obtained results. A better understanding of random searching processes may help to develop random-walk models with sound explicative power, sensu Ginzberg and Jensen (2004). This knowledge could clarify how animals face environmental uncertainty and reduced perceptual capabilities in large-scale displacements (Lima and Zollner 1996). Further, a solid relationship between animal behavior and the statistical properties of movement could be established, thus uncovering useful links between the behavioral (Bell 1991) and the pattern-based approaches common in spatial ecology (Okubo 1980, Tilman and Kareiva 1997).

Although some theoretical studies have already shown the potential role of CRWs and LWs in the understanding of animal random search strategies (Zollner and Lima 1999, Viswanathan et al. 1999), two fundamental questions about CRW and LW models still need to be addressed. First, quantifying their efficiency as random search strategies based on their respective statistical and scaling properties. Second, developing adequate biological interpretations of such properties in a random search context. The present contribution is a first effort to clarify the above points. For doing so, we have structured our analysis as follows. First, we demonstrate quantitatively relevant differences in the statistical properties of CRWs and LWs. Then, we discuss how such properties explain the different efficiencies obtained when the models are used as random search strategies in the ecological context. Finally, we suggest how the present results may lead to a better theoretical understanding of some fundamental aspects of large-scale animal displacements in real ecosystems. We would like to emphasize that our goal is not to provide recipes to analyze specific empirical data and determine which models would lead to a better fitting in a particular case. Instead, our purpose is to provide general criteria to evaluate why we should expect one of the models to fit better. Providing explicative power to random-walk models is especially necessary if such models are going to be used as null models, as well as if deviations from such null models are going to be interpreted biologically.

\section{METHODS}

Random walks constitute probabilistic discrete step models that involve strong simplifications of real animal movement behavior. In relation to more complex behavioralist models including many parameters, random-walk models ultimately express behavioral minimalism (Lima and Zollner 1996, Turchin 1998). Their main basic assumption holds that real animal movements consist of a discrete series of displacement events (i.e., move lengths) separated by successive reorientation events (i.e., turning angles). Discretization of complex movement behaviors will determine (after a large enough number of successive moves) the statistical distribution of displacement lengths on the one hand, and the statistical distribution of changes of direction (i.e., turning angles) on the other hand. From successive random draws of such distributions, we can obtain different movement path realizations. All the paths obtained by this method have statistical equivalence.

\section{The models}

We have used three random-walk models in our quantitative analysis. Correlated random walks (CRWs), Lévy walks (LWs), and a new model based on the previous ones which we have named Lévy-modulated correlated random walks (LMCRWs). Each model controls the directional persistence of the movement (i.e., the degree of correlation in the random walk) in a different way. Below we briefly discuss each model and the simulation procedures, leaving to the Appendix all the technical details.

CRW models combine a Gaussian (or other exponentially decaying) distribution of move lengths (i.e., displacement events) with a nonuniform angular distribution of turning angles (i.e., reorientation events). These models control directional persistence (i.e., the degree of correlation in the random walk) via the probability distribution of turning angles. In our study, we have used a wrapped Cauchy distribution (WCD [Batschelet 1981, Haefner and Crist 1994]) for the turning angles. Directional persistence is controlled by changing the shape parameter of the $\operatorname{WCD}(\rho)$. For $\rho=0$, we obtain a uniform distribution with no correlation between successive steps, thus Brownian motion 

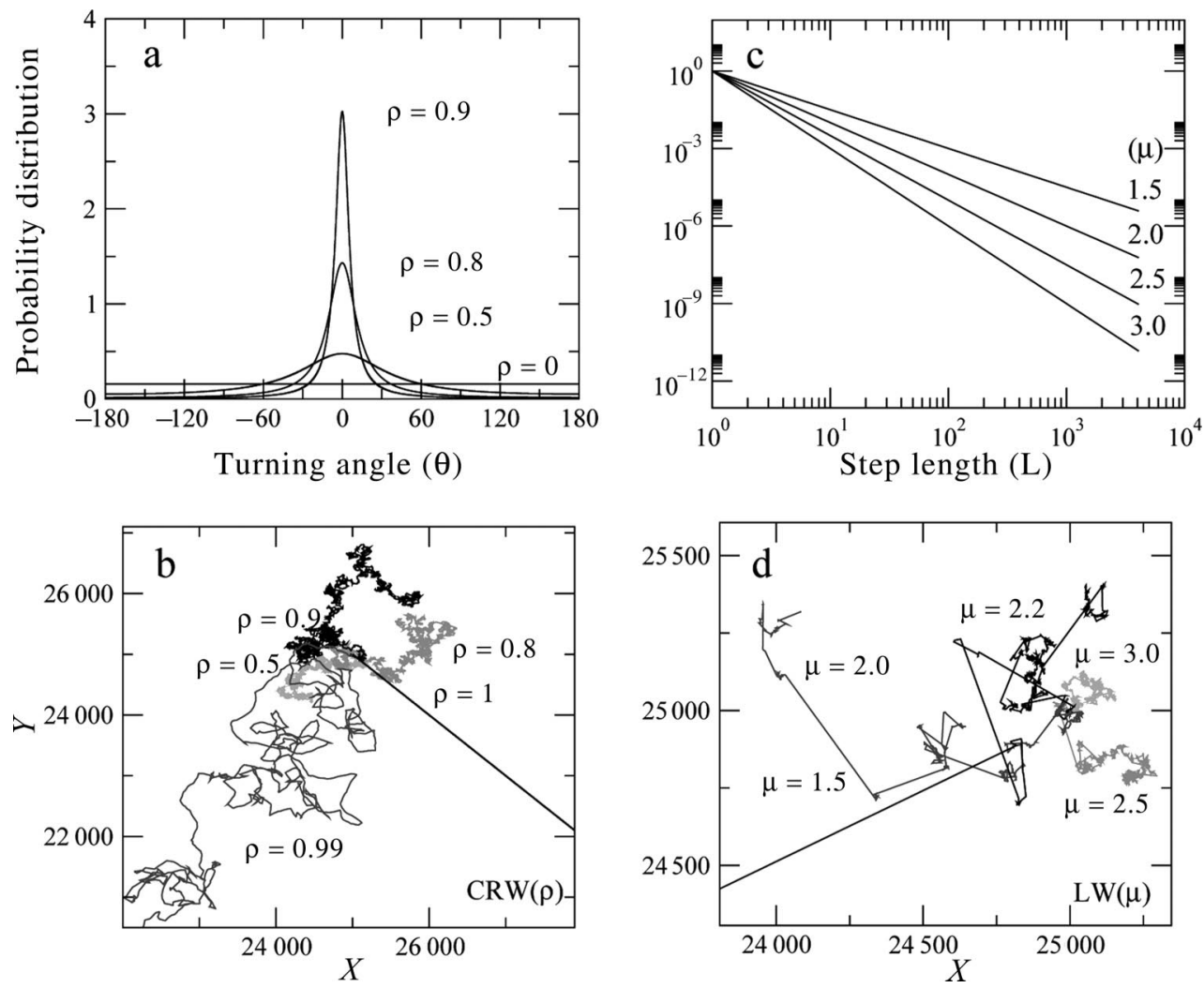

FIG. 1. (a) Shape of the wrapped Cauchy distribution used in the correlated random walk, for different values of the shape parameter $\rho$. (b) Examples of correlated random walks, generated by wrapped Cauchy distributions with different shape parameters. (c) Power-law distributions used in the Lévy walk, for different values of the Lévy exponent $\mu$. (d) Examples of Lévy walks, generated by power-law distributions with different Lévy exponents.

emerges. For $\rho=1$, we get a delta distribution at $0^{\circ}$ (Fig. 1a), leading to straight-line searches (Fig. 1b).

LW models involve a uniform distribution for the turning angles, but a power-law distribution for the move lengths (i.e., the so-called flights). The exponent of the power-law is named the Lévy index $(1<\mu \leq$ 3, see Fig. 1c) and controls the range of correlations in the movement. LW models thus comprise a rich variety of paths ranging from Brownian motion $(\mu \geq$ 3) to straight-line paths $(\mu \rightarrow 1$; Fig. 1d).

Finally, the LMCRW model introduced here generates a random walk with (i) a WCD for the turning angles within a flight, (ii) a Gaussian distribution of move steps within a flight, (iii) a uniform distribution for the turning angles between flights, and (iv) a powerlaw distribution of flight lengths. As in the LW model, the directional persistence of LMCRW is also introduced through a power-law distribution of move lengths (i.e., flights) but we can also modulate or control the degree of directional persistence during flight lengths through a WCD of turning angles (i.e., by changing the value of $\rho$ ). This new model can reveal which type of directional persistence controls the optimization of random searches, whether the power-law distribution of move lengths or the WCD of turning angles.

\section{The simulations}

The statistical properties of random-walk models should be evaluated at the long-term limit (i.e., large spatial scales and long temporal scales). When running simulations, this means that both the turning angle and the move length probability distributions should be thoroughly sampled (i.e., this is especially important with long-tailed probability distributions). The longterm statistical properties of random searches only emerge once a minimum amount of time and space are included in the search. The spatiotemporal scales required for that are not fixed, but are organism specific.

A first group of simulations studied the behavior of a relevant macroscopic property of random walks: the mean square displacement (msd), defined as the squared distance that an organism moves from its starting location to another point during a given time, averaged over many different random walkers. Msd is related to the CRW metric of net squared displacement but is not exactly the same (see the Appendix for more details). In this set of simulations, we computed the 
msd for a set of random walkers moving in a twodimensional arena at different times considering different parameter values for $\rho$ and $\mu$ in CRWs and LWs, respectively.

We devised a second group of simulations in order to determine the search efficiencies $(\lambda \eta)$ of the three types of random walks (i.e., CRW, LW, and LMCRW models). The objects that are looked for are called targets. In general, a target may represent any important resource for a searcher (i.e., food, mates, breeding habitats, nesting sites, etc.). In our simulations, targets are nonmobile, thus we prefer the term target sites (e.g., static resources, suitable habitats, etc.). We defined the search efficiency function $\eta$ as the ratio of the number of target sites visited to the total distance traversed by the searcher. Note that in LWs, $\eta=\eta(\mu)$, in CRWs, $\eta$ $=\eta(\rho)$, and in the LMCRWs, $\eta=\eta(\mu, \rho)$. Specifically, the simulations quantified the average search efficiency of a set of random walkers provided with a radial detection distance $r_{\mathrm{d}}$, that looked for nonmobile circular items with radius $r_{\mathrm{t}}$ (i.e., target sites) in a two-dimensional space with periodic boundary conditions. Target sites were uniformly distributed in an otherwise homogeneous arena. The scaling of the search scenarios is based on a unique key parameter: the mean free path $(\lambda)$, which is defined as the average distance between two target sites. The mean free path is inversely related to the density of target sites and the searcher's detection radius and gives us the idea of how far the searcher moves before "detecting" a target (see Appendix). We defined three different search scenarios with increasing values of $\lambda$ representing a decreasing gradient of target site densities (we kept the same searcher's detection radius for the three search scenarios). To represent different search strategies, we ran the simulations using different parameter values for each random-walk model (i.e., LW and CRW). The product $\lambda \eta$ allows us to obtain a metric for the search efficiency that is independent of the target site density.

We considered two kinds of encounter dynamics in the efficiency simulations: destructive and nondestructive. In the case of nondestructive searches, the searcher can visit the same target site many times. This accounts for those cases in which target sites become only temporarily depleted or searchers become satiated and leave the area. In the case of destructive searches, the target site found by the searcher becomes undetectable in subsequent displacements - the target site "disappears." In this case, just to make averages always with the same target density, we generated a new target site at random in the searching space. Both types of encounter dynamics may represent real ecological situations and should demand different random-search strategies in order to optimize the rate of encounters (Viswanathan et al. 1999). The nondestructive and destructive searching scenarios represent the limit cases of a continuum of possible target regeneration dynamics (Raposo et al. 2003). Moreover, the nondestructive case with uniformly distributed targets bears a similarity to a destructive case with patchy or fractal targetsite distributions (Viswanathan et al. 1999). Thus, these simulations cover a wide range of natural searching situations.

\section{Results}

\section{On the macroscopic properties of CRWs and LWs}

Random-walk theory assumes that a particularly relevant macroscopic property of random walks involves the scaling in relation to time of the mean square displacement (msd) of the diffusing organisms: $\left\langle R(t)^{2}\right\rangle \sim$ $t^{\alpha}$, where $\alpha$ characterizes the behavior of diffusive processes. In normal (i.e., Fickian) diffusive processes, the msd increases linearly with time $(\alpha=1)$. The simplest example of this is particles (or organisms) moving independently and executing uncorrelated random walks; i.e., pure Brownian motion. On the other hand, processes that lead to a nonlinear dependence of msd over time, known as anomalous diffusion, typically occur in complex or long-range correlated phenomena (Gefen et al. 1983). Anomalous diffusion arises due to longrange statistical dependence between steps in a random walk and can involve a subdiffusive $(\alpha<1)$ or a superdiffusive $(\alpha>1)$ process. The fastest possible superdiffusion occurs when particles (or organisms) execute unbroken straight-line paths corresponding to ballistic motion or dispersal with $\alpha=2$.

As stated above, CRW models control persistence (i.e., the degree of correlation in the random walk) via the probability distribution of turning angles. However, from the macroscopic point of view, CRWs represent simple Markovian processes that, by their very nature, cannot generate long-range correlations in the movement (Johnson et al. 1992). Thus, for CRWs, the msd can depart from the linear increase with time only over a particular range of temporal and spatial scales, but, at the long-term limit, the relation always becomes linear. Therefore, at the long-term limit, CRWs models appear like uncorrelated random walks: they can only give rise to Brownian motion. Fig. 2a shows the behavior of the msd in a CRW as we vary the shape parameter $\rho$ of the WCD (used to correlate the steps). For any value $\rho<1$ (even for values close to 1 , e.g., $\rho=0.95)$ the macroscopic behavior of movement converges rapidly $(t \approx 100$ ) to the Brownian motion domain. Only for the limit case of $\rho=1$ do we obtain a ballistic motion. Thus, there is no a smooth way to go from Brownian to ballistic motion by changing the turning angle distribution parameter of CRWs $(\rho)$. Instead, only two macroscopic motions emerge in the long term limit: pure Brownian $(\rho<1)$ or ballistic dispersal behavior $(\rho=1)$.

When persistence arises through a power-law distribution of move lengths instead of Markovian shortrange angle correlations, a new property emerges because of long-range move length correlations. A grad- 

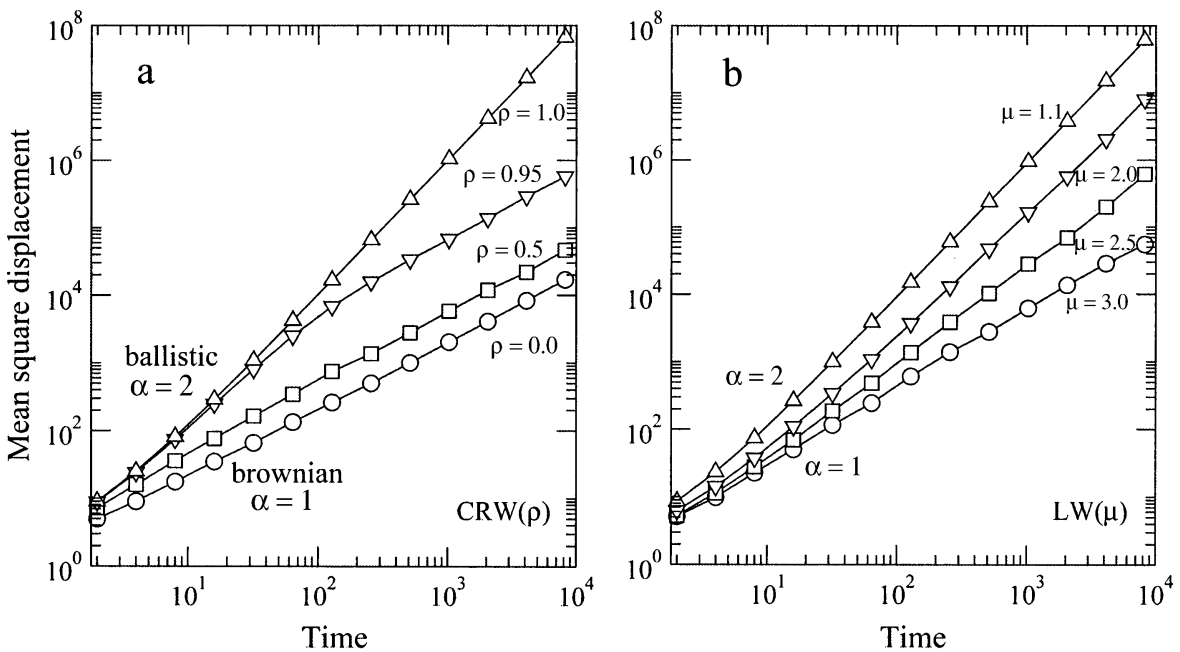

FIG. 2. Mean square displacement (msd), obtained by averaging 500 individuals, in relation to time for (a) correlated random walks and (b) Lévy walks. The scaling behavior indicates short $(\alpha=1)$ and long-range $(\alpha>1)$ correlations in the random walks; $\alpha=2$ indicates ballistic (i.e., straight-line) motion.

ual change in the Lévy exponent (i.e., $\mu$ ) corresponds to a gradual change in the diffusivity (i.e., $\alpha$ ) that does not vanish at the long-term limit. A gradual transition from normal diffusion ( $\alpha=1$ for $\mu \geq 3$ ) to ballistic motion $(\alpha \rightarrow 2$ for $\mu \rightarrow 1$ ) becomes possible for LWs (Fig. 2b). Therefore, different Lévy exponents of the power-law distribution of move lengths provide a whole variety of super-diffusive behaviors $(1<\alpha<$ 2 for $1<\mu<3$ ). Thus, changing the Lévy exponent implies a qualitative change in the macroscopic and long-term properties of the movement as a whole.

\section{On the search efficiency of the random-walk models}

Fig. 3 shows the changes in the searching efficiency measured as $\lambda \eta$ of both a CRW $(\eta(\rho))$ and a LW $(\eta(\mu))$ when varying the parameters controlling the degree of persistence in the walk. We have considered three search scenarios $(\lambda=100,1000$, and 5000 [see Methods and Appendix]) representing a decreasing gradient from high to low target densities, and two encounter dynamics: destructive and nondestructive.

In all cases, LWs are more efficient than CRWs. As density diminishes (i.e., $\lambda$ increases), LWs become even more efficient (than CRWs) in both dynamical types of searches, but with different optimal Lévy exponents $\left(\mu_{\mathrm{opt}}\right)$. In the destructive case, $\mu_{\mathrm{opt}} \rightarrow 1$ and, in the nondestructive case, $\mu_{\mathrm{opt}} \approx 2$. These results agree with previous works on Lévy random-walk searches (Viswanathan et al. 1996, 1999). Note the convergence of CRWs $(\rho=0)$ with LWs $(\mu=3)$ and CRW $(\rho=$ $1)$ with LW $(\mu \rightarrow 1)$. In the former, both models correspond essentially to Brownian motion, whereas, in the latter, they give rise to straight-line motion (i.e., ballistic dispersal behavior). The relevant differences appear precisely in the transition from Brownian to ballistic motion. Within the whole range of possible random walks from the Brownian (pure random walk) to the ballistic (straight-line walk), searchers performing LWs exhibit higher efficiency than searchers performing CRWs in the long-term encounter statistics (i.e., $\eta\left(\mu_{\text {opt }}\right) \geq \eta\left(\rho_{\text {opt }}\right)$ in Fig. 3).

In destructive searches (Fig. 3), revisiting target sites penalizes the search efficiency because targets are consumed. Therefore, the larger the persistence in the movement, the larger the search efficiency. Persistence increases with increasing $\rho$ in CRWs and decreasing $\mu$ in LWs. However, changes in Lévy exponent not only modify short-term persistence of the walk but also involve concomitant changes in the macroscopic properties of the movement that the CRWs do not have. As $\mu$ decreases, superdiffusivity of movement is enhanced (see Fig. 2b). Superdiffusion increases the efficiency beyond short-ranged persistence; that is why LWs are more efficient than CRWs in destructive searches.

In nondestructive searches (Fig. 3), revisiting sites is not penalized because targets are not consumed. Therefore, persistence and superdiffusivity do not influence search efficiency significantly. Indeed, they are useful to avoid empty areas created by destructive encounter dynamics. This fact explains why the efficiency of CRWs in the nondestructive case is $\rho$ independent. However, the higher values for the LWs efficiency (which furthermore remains dependent on $\mu$ ) may be due to another particular LW property not shared with CRWs, namely, scale invariance. Thus, our results clearly show that scale invariance plays a crucial role in optimizing encounter rates in the nondestructive cases.

Fig. 4 shows the searching efficiency measured as $\lambda \eta$ for the LMCRW model $\eta(\mu, \rho)$ and $\lambda=5000$ in destructive and nondestructive searches. Changes in the $\rho$ and $\mu$ parameters account for different searching 
Destructive search
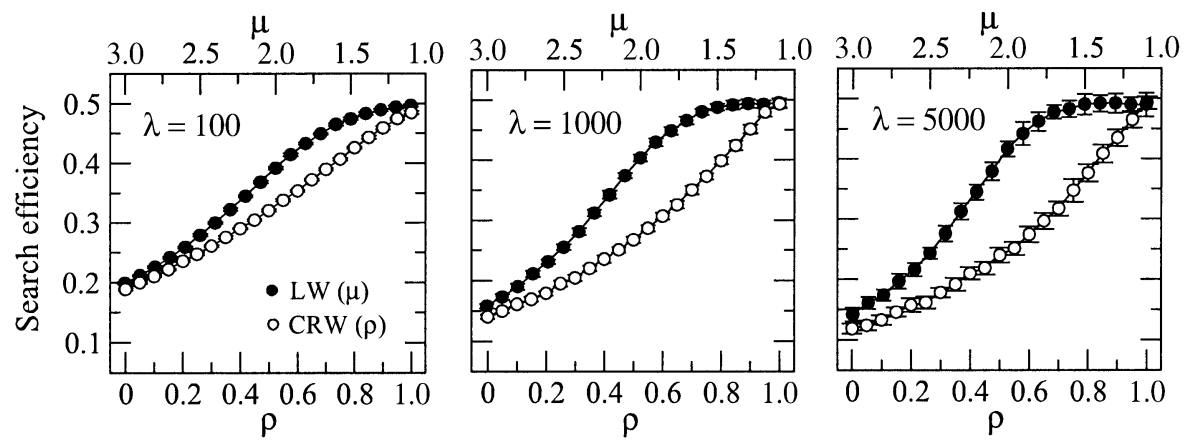

Nondestructive search
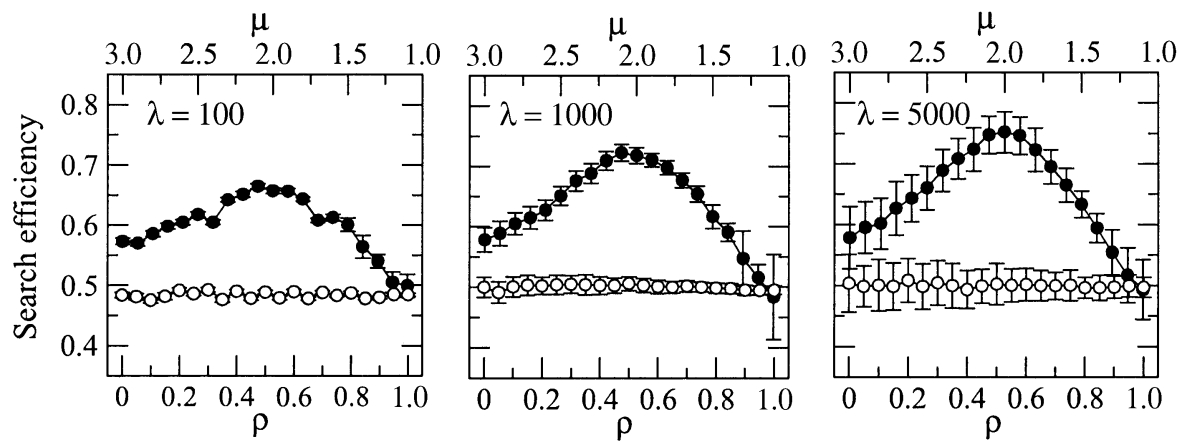

FIG. 3. Comparison of the search efficiency (measured as $\lambda \eta$ ) of different animal search strategies. The panels assume three different target densities $(\lambda=100,1000$, and 5000, respectively). The three top panels assume that the targets are destroyed once found, whereas the three bottom panels assume that the targets are not destroyed. Search efficiency is plotted as a function of the shape parameter $\rho$ for correlated random walks (open circles) and as a function of the Lévy exponent $\mu$ for Lévy walks (solid circles). Error bars represent \pm SD.

strategies with different searching efficiencies. For the destructive case, $\rho$ must be very close to 1 (i.e., move lengths must be straight lines) in order to get optimal searches. However, for the nondestructive case, a certain degree of sinuosity during move lengths (i.e., 0.8 $<\rho<1$ ) does not diminish the searching efficiency considerably. In nondestructive searches, as the degree of persistence within flights diminishes, the overall efficiency of the search diminishes and the Lévy index giving rise to optimal searching strategies also decreases.

The LMCRW model results for nondestructive dynamics (Fig. 4) show that reorienting the movement at power-law time intervals have more influence in the search efficiency than small direction deviations during flights. This explains why LWs appear robust in their efficiency even with sinuous flights due to the embedded CRWs. However, persistence within flights cannot fall too low, (e.g., a proper range $0.8<\rho<1$ ), otherwise the pattern of move lengths will lose fractality (i.e., scale invariance) due to the highly sinusoidal and looping paths, and so no pure LWs will arise at the necessary scales. Note that, for $\rho<1$, a natural upper cutoff appears for the scale invariance, due to the correlation length of the embedded CRW, and thus superdiffusive behavior does not occur in LMCRWs.

\section{DISCUSSION}

Most ecological interactions must necessarily begin with a physical encounter (i.e., sensu strictu), which usually takes place after an active or passive searching process. By a search, we mean the process of looking for the presence of real or suspected objects of interest (i.e., food, mate, shelter, etc.) which we call "targets." Random search strategies can only exist when there is some degree of uncertainty in the behavior of targets. When there is no search uncertainty, because both spatial and temporal behavior of targets are known (Garber 1988) or because displacements are dictated by strong external cues (Hauser et al. 1975), the resulting animal movement cannot be considered a search. Those situations where the overall animal movement is mainly driven by strong internal navigation mechanisms (i.e., migrations) or environmental constraints (i.e., strong physical barriers) should also not be considered a search.

In any interactive process between individuals, the search is only one component among others. In foraging strategies, we also may consider the handling times, pursuit costs, predation risks, discount decisions, prey selection, etc. These components can be subjected to optimization by natural selection, the search com- 

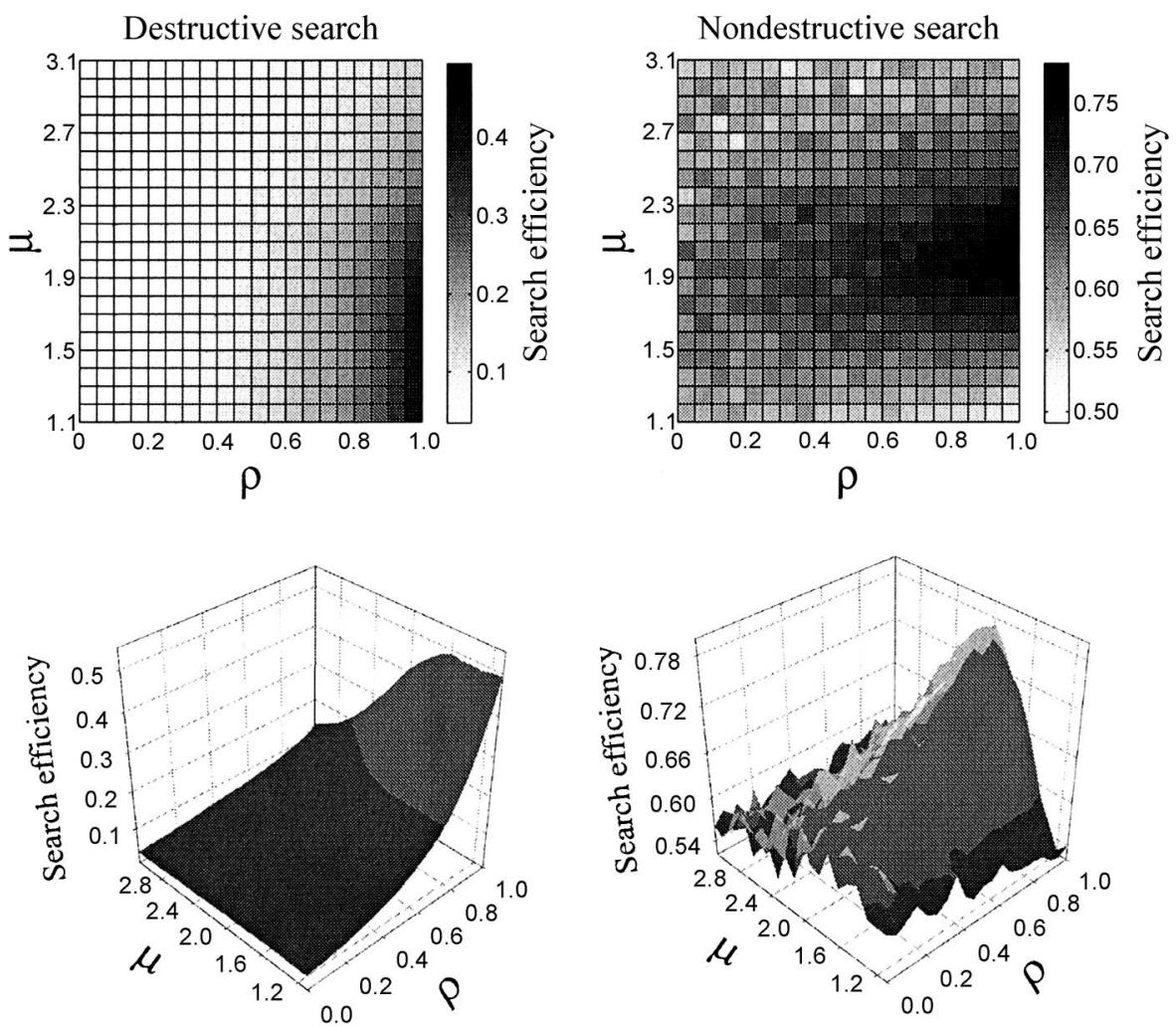

FIG. 4. Search efficiency (measured as $\lambda \eta$ ) of the Lévy-modulated correlated random walk, plotted as a function of the shape parameter $\rho$ and the Lévy exponent $\mu$. The graphs assume a target density of $\lambda=5000$. Results are shown for destructive and nondestructive encounter dynamics.

ponent being more or less relevant depending on the cognitive capacities of organisms and the predictability of its environment. The optimization of a search strategy involves the selection of a specific set of "rules of search" that enhances the probability of finding unknown located items.

\section{Systematic and random search strategies}

According to the characteristics of the "rules of search" we can classify the continuum of search strategies in two main types: systematic and random searches. In systematic searches, the rules to optimally cover a given area are based on deterministic algorithms (i.e., fixed and organized plans), while, in random searches, these rules rely on stochastic processes (i.e., the sampling of probability distributions). Systematic search strategies only work when some a priori relevant (although partial) information about target characteristics or locations at "landscape level" is available. The Archimedean spirals represent one of the most common systematic searching rules in homing behaviors (Bell 1991, Turchin 1998). As available information regarding positional, kinetic, or behavioral characteristics of targets decreases, systematic searches become less effective. In these situations, animals must attempt to move in such a way so as to optimize their chances of locating resources by increasing "the chances of covering certain regions," and thus, different optimal solutions arise by merely embracing different random strategies (Viswanathan et al. 1999, Bartumeus et al. 2002a, b, Raposo et al. 2003, Santos et al. 2004). An interesting example that illustrates a switch between the two types of search is provided by the homing behavior of a desert isopod (Hoffmann 1983a,b). If an isopod misses the entrance of its burrow by a few millimeters, it must search for the entrance. If the excursion has covered only a short distance, it searches following an Archimedean spiral pattern. If the burrow is not found during this initial phase, the animal extends its range, and moves in broad loops and meanders, returning repeatedly to the starting point of the search (Hoffmann 1983a). Therefore, when pure systematic search fails, the isopod uses a mixture of systematic (i.e., returns to the starting point) and random (i.e., meandering) search.

The opposite case, the shift from random search strategies to systematic searches, should reflect an increase in the predictability of the spatiotemporal behavior of targets. Foraging scenarios involving nondestructive searches may enhance the incorporation of systematic rules in relation to the spatial and temporal pattern of rewards (learned after repeated visits to the 
targets). This is the case of trapline foraging in bumble bees (Thomson 1996, Thomson et al. 1997, Williams and Thomson 1998). Hermit hummingbird traplining also includes systematic "rules of thumb" to directly interfere with the search success of competitors (Gill 1988). In this case, uncertainty arises in relation to how the others behave, and search rules relaying on stochastic processes may become an option (particularly for those individuals repeatedly "failing" in their interactions). Therefore, in any given environment, there might be a range of search strategies that can be successful, and individuals may differ in the search strategy used. As is recognized by authors, the extension to what learned systematic search mechanisms work in more complex situations remains uncertain (Gill 1988, Williams and Thomson 1998).

So far, animal search rules relying on stochastic processes are not considered in behavioral evolution because it is assumed that sensorial or cognitive improvements override the need of random search in nature. However, it should be considered that, (i) in some search processes, a high degree of uncertainty is unavoidable, and (ii) in such scenarios, the success of the search can be improved by optimizing random search strategies.

\section{Lévy walks and correlated random walks}

The key assumptions of CRWs involve the presence of directional persistence at certain scales. However, random-walk models with short-range correlations, such as CRWs, converge to a Brownian motion (i.e., normal diffusive process) at certain time scales, not very long (Fig. 2a). Therefore, at large enough spatiotemporal scales when persistence breaks down, the macroscopic statistical properties of CRWs become the same as in uncorrelated random walks. This fact, which is part of the standard random-walk theory, has been used to justify the usage of uncorrelated random walks and normal diffusion models in ecology (Okubo 1980, Berg 1983). However, our results show that their properties do not allow the best optimal random searches if large enough spatiotemporal scales are of relevance. This might be the reason that other biological considerations such as dispersal risks or energetic costs are needed in order to obtain optimal solutions when these models are used as random search strategies (Cain 1985, Zollner and Lima 1999). Indeed, these studies concluded that without biological constraints any random search strategy should be equally efficient. Our results show that this is not the case.

The key assumptions of LWs involve superdiffusive and scale-invariant phenomena and ensure (1) departures from normal diffusion at all scales (Fig. 2b), and (2) the possibility of optimizing encounter rates just by pure statistical physics considerations in a wide range of different searching scenarios (Fig. 3). Commonly, departures from normal diffusion have been attributed to (i) "large-scale-oriented" movements of in- dividuals (e.g., seasonal migration, landscape cues, cognitive maps, etc.), or (ii) physical barriers or corridors in the landscape that alter an organism's otherwise random movement (Johnson et al. 1992). However, to exploit the statistical advantages provided by superdiffusion and scale invariance, it is not necessary to assume cognitive mapping or high spatial memory capacities of organisms (as some recent models suggest [Gautestad and Mysterud 2005]). Instead, simple "rules of thumb" in accordance with stochastic laws may be enough. Consistently, scale-invariant animal movement is a widespread phenomenon in nature, observed from microorganisms to large vertebrates. Thus far, Lévy walk patterns have been observed in soil amoebas (Levandowsky et al. 1997), planktonic organisms (Bartumeus et al. 2003), bumble bees (Heinrich 1979), seabirds (Viswanathan et al. 1996), large terrestrial herbivores (Marell et al. 2002), social cannids (Atkinson et al. 2002), arboreal primates (RamosFernández et al. 2004), and Arctic seals (Austin et al. 2004).

\section{Lévy walks as adaptive random-search strategies}

We can inquire whether situations where random strategies are the optimal solutions to a search problem occur with sufficient frequency (at least to significantly modify the fitness of the populations). And, if so, whether there are specific biological mechanisms on which natural selection could impinge to develop specific behavioral traits. Biological mechanisms optimizing the "chances of finding" unknown located items should not necessarily be the same as those allowing for the "detection" of such items. Probably, the selective pressures and the triggering stimuli are different in each case, and the combination of both mechanisms can provide a huge behavioral plasticity to adapt searches to widely different ecological scenarios.

Based on the standard theory of random walks (Okubo 1980, Berg 1983), it seems reasonable to think that selective pressure on "local scanning mechanisms", may not influence the statistical macroscopic properties of the walk, although it can provide short-range correlations with the statistical properties of CRWs. In contrast, selective pressure on "episodic reorientation mechanisms" could modify these macroscopic properties, and therefore change the chances of finding unknown located items. Our quantitative results comparing LW and CRW searching efficiencies show that the optimization of random searches mainly depends on the optimal temporal execution of reorientation events (Fig. 3). Even when directional persistence within displacements is not really high, the temporal execution of reorientation events is the key factor controlling the main properties of the random walk (Fig. 4). This result supports previous studies demonstrating the robustness of LWs against short-scale "memory effects" (da Luz et al. 2001, Raposo et al. 2003). Therefore, random search strategies are not incompatible with short-scaled 
(in time or space) "memory effects" (i.e., local scanning mechanisms, systematic "rules of thumb," etc).

Overall, we suggest that scale-free punctuations in animal movement (i.e., stops, strong reorientations, behavioral distinctive interruptions during the walk, etc.) could be the basis for a stochastic organization of the search at landscape level. Some preliminary empirical results point towards this hypothesis (Bartumeus et al. 2003). The heterotrophic dinoflagellate Oxyrrhis marina switches from an exponential to an inverse square power-law distribution of flight times (that is, from diffusive to superdiffusive behavior) when its prey Rhodomonas sp. decreases in abundance. The specific biological mechanism involved in this searching behavioral change are transient arrests of the longitudinal flagellum beat, which are observable by simple visual inspection of the animal's movement. Thus, continuous helical motion is interrupted by sudden changes in direction (i.e., reorientation leaps) that govern the longterm searching walk of this planktonic predator. Although Lévy walk patterns have been identified for a wide variety of organisms (Viswanathan et al. 1996, Levandowsky et al. 1997, Atkinson et al. 2002, Marell et al. 2002, Austin et al. 2004, Ramos-Fernández et al. 2004), the case study of Oxyrrhis marina is so far the only example where the biological mechanism generating a Lévy-type walk has been elucidated. The biological mechanisms generating Lévy walk patterns in other species remain to be investigated. In this sense, two questions should be answered first: Are these patterns caused by a random search strategy or have they emerged from complex behavioral processes, external drivers, and so forth? Can we identify reorientation mechanisms within animal behavioral traits? We expect that further investigations about when and how organisms actively discretize their movements (Kramer and McLaughlin 2001, O'Brien et al. 1990) will facilitate the finding of adaptive mechanisms capable of optimizing random search statistics.

\section{ACKNOWLEDGMENTS}

F. Bartumeus thanks D. Alonso and P. Fernández for contributing in the first stages of analytical and numerical approaches to Lévy walk simulations. F. Bartumeus also thanks R. V. Solé and the Complex System Lab group for inspiring talks on Lévy walks and random searches. G. M. Viswanathan and M. G. E. da Luz thank E. Raposo for fruitful discussions. Also CNPq and FAPEAL (G. M. Viswanathan), and CNPq, CT-Infro, CT-Energ, Capes, and Fundação Araucária (M. G. E. da Luz) are acknowledged for research grants.

\section{Literature Cited}

Atkinson, R. P. D., C. J. Rhodes, D. W. Macdonald, and R. M. Anderson. 2002. Scale-free dynamics in the movement patterns of jackals. Oikos 98:134-140.

Austin, D., W. D. Bowen, and J. I. McMillan. 2004. Intraspecific variation in movement patterns: modeling individual behavior in a large marine predator. Oikos 105:15-30.

Bartumeus, F., J. Catalan, U. L. Fulco, M. L. Lyra, and G. M. Viswanathan. 2002a. Optimizing the encounter rate in biological interactions: Lévy vs. Brownian strategies. Physical Review Letters 88: article 097901.
Bartumeus, F., J. Catalan, U. L. Fulco, M. L. Lyra, and G. M. Viswanathan. 2002b. Optimizing the encounter rate in biological interactions: Lévy vs. Brownian strategies [Phys. Rev. Lett. 88, 097901 (2002)]. Physical Review Letters 89: article 109902(E).

Bartumeus, F., F. Peters, S. Pueyo, C. Marrasé, and J. Catalan. 2003. Helical Lévy walks: Adjusting searching statistics to resource availability in microzooplankton. Proceedings of the National Academy of Sciences (USA) 100:1277112775.

Batschelet, E. 1981. Circular statistics in biology. Academic Press, New York, New York, USA.

Bell, W. J. 1991. Searching behaviour: the behavioural ecology of finding resources. Cambridge University Press, Cambridge, UK.

Benhamou, S. 2004. How to reliably estimate the tortuosity of an animal's path: straightness, sinuosity, or fractal dimension? Journal of Theoretical Biology 229:209-220.

Berg, H. C. 1983. Random walks in biology. Princeton University Press, Princeton, New Jersey, USA.

Bergman, C., J. A. Schaefer, and S. N. Luttich. 2000. Caribou movement as a correlated random walk. Oecologia 123: 364-374.

Bovet, J., and P. Bovet. 1993. Computer-simulations of rodent homing behaviour, using a probabilistic model. Journal of Theoretical Biology 161:145-156.

Bovet, P., and S. Benhamou. 1988. Spatial analysis of animals' movements using a correlated random walk model. Journal of Theoretical Biology 131:419-433.

Bovet, P., and S. Benhamou. 1991. Optimal sinuosity in central place foraging movements. Animal Behaviour 42:5762.

Cain, M. L. 1985. Random search by herbivorous insects in a simulation model. Ecology 66:876-888.

Crist, T. O., D. S. Guertin, J. A. Wiens, and B. T. Milne. 1992. Animal movement in heterogeneous landscapes: an experiment with Elodes beetles in shortgrass prairie. Functional Ecology 6:536-544.

da Luz, M. G. E., S. V. Buldyrev, S. Havlin, E. P. Raposo, H. E. Stanley, and G. M. Viswanathan. 2001. Improvements in the statistical approach to random Lévy flight searches. Physica A 295:89-92.

Fritz, H., S. Said, and H. Weimerskirch. 2003. Scale-dependent hierarchical adjustments of movement patterns in a long-range foraging seabird. Proceedings of the Royal Society of London Series B 270:1143-1148.

Garber, P. A. 1988. Foraging decisions during nectar feeding by tamarin monkeys (Saguinus mystax and Saguinus fusicollis, Callitrichidae, Primates) in Amazonian Peru. Biotropica 20:100-106.

Gautestad, A. O., and I. Mysterud. 2005. Intrinsic scaling complexity in animal dispersion and abundance. American Naturalist 105:44-56.

Gefen, Y., A. Aharony, and S. Alexander. 1983. Anomalous diffusion on percolating clusters. Physical Review Letters 50:77-80.

Gill, F. B. 1988. Trapline foraging by hermit hummingbirds: competition for an undefended, renewable resource. Ecology 69:1933-1942.

Ginzberg, L. R., and C. X. J. Jensen. 2004. Rules of thumb for judging ecological theories. Trends in Ecology and Evolution 19:121-126.

Haefner, J. W., and T. O. Crist. 1994. Spatial model of movement and foraging in harvester ants (Pogonomyrex) (I): The role of memory and communication. Journal of Theoretical Biology 166:299-313.

Hauser, D. C. R., M. Levandowsky, and J. Glassgold. 1975. Ultrasensitive chemosensory by a protozoan to epinephrine and other neurochemicals. Science 190:285-286. 
Heinrich, B. 1979. Resource heterogeneity and patterns of movement in foraging bumblebees. Oecologia 40:235-245.

Hoffmann, G. 1983a. The random elements in the systematic search behavior of the desert isopod Hemilepistus reaumuri. Behavioural Ecology and Sociobiology 13:81-92.

Hoffmann, G. 1983b. The search behavior of the desert isopod Hemilepistus reaumuri as compared with a systematic search. Behavioural Ecology and Sociobiology 13:93-106.

Johnson, A. R., B. T. Milne, and J. A. Wiens. 1992. Diffusion in fractal landscapes: simulations and experimental studies of tenebrionid beetle movements. Ecology 73:1968-1983.

Kareiva, P. M. and N. Shigesada. 1983. Analyzing insect movement as a correlated random walk. Oecologia 56:234238.

Klafter, J., M. F. Shlesinger, and G. Zumofen. 1996. Beyond Brownian motion. Physics Today 49:33-39.

Klafter, J., B. S. White, and M. Levandowsky. 1989. Microzooplankton feeding behavior and the Lévy walk. Pages 281-296 in G. Hoffmann and W. Alt, editors. Biological motion. Springer-Verlag, Heidelberg, Germany.

Kramer, D. L., and R. L. McLaughlin. 2001. The behavioral ecology of intermittent locomotion. American Zoologist 41:137-153.

Levandowsky, M., J. Klafter, and B. S. White. 1988a. Feeding and swimming behavior in grazing zooplankton. Journal of Protozoology 35:243-246.

Levandowsky, M., J. Klafter, and B. S. White. 1988b. Swimming behavior and chemosensory responses in the protistan microzooplankton as a function of the hydrodynamic regime. Bulletin of Marine Science 43:758-763.

Levandowsky, M., B. S. White, and F. Schuster. 1997. Random movements of soil amoebas. Acta Protozoologica 36: 237-248.

Lima, S. L., and P. A. Zollner. 1996. Towards a behavioural ecology of ecological landscapes. Trends in Ecology and Evolution 11:131-135.

Mandelbrot, B. B. 1977. Fractals: form, chance and dimension. Fremman, San Francisco, California, USA.

Marell, A., J. P. Ball, and A. Hofgaard. 2002. Foraging movement paths of female reindeer: insights from fractal analysis, correlated random walks, and Lévy flights. Canadian Journal of Zoology 80:854-865.

Metzler, R., and J. Klafter. 2004. The restaurant at the end of the random walk: recent developments in the description of anomalous transport by fractional dynamics. Journal of Physics A: Mathematics and General 37:R161-R208.

O'Brien, W. J., H. I. Browman, and B. I. Evans. 1990. Search strategies of foraging animals. American Scientist 78:152160.

Okubo, A. 1980. Diffusion and ecological problems: mathematical models. Springer-Verlag, Berlin, Germany.

Ramos-Fernández, G., J. L. Mateos, O. Miramontes, G. Cocho, H. Larralde, and B. Ayala-Orozco. 2004. Lévy walk patterns in the foraging movements of spider monkeys (Ateles geoffroyi). Behavioural Ecology and Sociobiology 55: 223-230.

Raposo, E. P., S. V. Buldyrev, M. G. E. da Luz, M. C. Santos, H. E. Stanley, and G. M. Viswanathan. 2003. Dynamical robustness of Lévy search strategies. Physical Review Letters 91:2-4.

Santos, M. C., E. P. Raposo, G. M. Viswanathan, and M. G. E. da Luz. 2004. Optimal random searches of revisitable targets: crossover from superdiffusive to ballistic random walks. Europhysics Letters 67:734-740.

Shlesinger, M. F., and J. Klafter. 1986. Lévy walks versus Lévy flights. Pages 279-283 in H. E. Stanley and N. Ostrowski, editors. On growth and form. Martinus Nijhof Publishers, Amsterdam, The Netherlands.

Shlesinger, M. F., G. Zaslavsky, and U. Frisch, editors. 1995. Lévy flights and related topics in physics. Springer-Verlag, Berlin, Germany.

Thomson, J. D. 1996. Trapline foraging by bumble bees: I. Persistence of flight-path geometry. Behavioral Ecology 7: 158-164.

Thomson, J. D., M. Slatkin, and B. A. Thomson. 1997. Trapline foraging by bumble bees: II. Definition and detection from sequence data. Behavioral Ecology 8:199-210.

Tilman, D., and P. Kareiva, editors. 1997. Spatial ecology: the role of space in population dynamics and interspecific interactions. Princeton University Press, Princeton, New Jersey, USA.

Turchin, P. 1991. Translating foraging movements in heterogeneous environments into the spatial distribution of foragers. Ecology 72:1253-1266.

Turchin, P. 1998. Quantitative analysis of movement: measuring and modelling population redistribution in animal and plants. Sinauer Associates, Sunderland, Massachusetts, USA.

Viswanathan, G. M., V. Afanasyev, S. V. Buldyrev, E. J. Murphy, P. A. Prince, and H. E. Stanley. 1996. Lévy flights search patterns of wandering albatrosses. Nature 381:413415.

Viswanathan, G. M., S. V. Buldyrev, S. Havlin, M. G. E. da Luz, E. P. Raposo, and H. E. Stanley. 1999. Optimizing the success of random searches. Nature 401:911-914.

Weeks, E. R., and H. L. Swinney. 1998. Anomalous diffusion resulting from strongly asymmetric random walks. Physical Review E 57:4915-4920.

Williams, N. M., and J. D. Thomson. 1998. Trapline foraging by bumble bees: III. Temporal patterns of visitation and foraging success at single plants. Behavioral Ecology 9: 612-621.

Zollner, P. A., and S. L. Lima. 1999. Search strategies for landscape-level interpatch movements. Ecology 80:10191030 .

\section{APPENDIX}

A detailed description of the random-walk models and simulation procedures is available in ESA's Electronic Data Archive: Ecological Archives E086-168-A1. 